\title{
A Geometric Programming Solution for the Mutual-Interference Model in HetNets
}

\author{
Luis Diez, George-Pantelimon Popescu and Ramón Agüero, Senior Member, IEEE
}

\begin{abstract}
It is well known that the use of Heterogeneous Networks and densification strategies will be crucial to handle the wireless cellular traffic increase that is foreseen in the forthcoming years. Hence, the scientific community is putting effort into the proposal and assessment of radio resource management solutions for this type of deployments. For that, an accurate modeling of the underlying resources is mandatory. In this letter we propose a mutual-interference model, which enables a precise estimation of the Signal to Interference and Noise Ratio (SINR), compared to the widespread constant-load alternative. This is of utter relevance, since the SINR has a direct influence on the spectral efficiency and, consequently, on the resources to be allocated. We also propose a transformation of the corresponding resource assignment problem, so that it can be solved using Geometric Programming techniques. The validity of this transformation is assessed by comparing the corresponding solution with the one that would have been obtained exploiting a heuristic approach (Simulated Annealing).
\end{abstract}

Index Terms-Network Model, HetNets, LTE, Mutual Interference, Geometric Programing

\section{INTRODUCTION}

During the latest years we have witnessed a huge increase of the mobile data traffic demand, and this trend is expected to remain in the near future [1]. In order to overcome the limitations of currently available technologies ( $3 \mathrm{G}$ and $4 \mathrm{G}$ ), forthcoming $5 \mathrm{G}$ systems are expected to support a thousandfold capacity increase [2]. Among the different techniques that are being studied in the framework of $5 \mathrm{G}$ related solutions, network densification stands out as one of the most attractive alternatives to appropriately deal with the expected traffic boost [3]. The deployment of ultra-dense networks will nonetheless require more complex resource management solutions and access policies, in order to ensure that the potential network capacity can be effectively exploited.

Several studies have already analyzed the performance of novel access techniques, such as Coordinated Multi-Point (CoMP) or the decoupling of uplink and downlink connections [4], as well as different access architectures, such as C-RAN [5]. Many of these studies have been conducted by

L. Diez and R. Agüero are with the University of Cantabria, Communications Engineering Department, Santander, Spain (email: \{ldiez,ramon\}@tlmat.unican.es)

G-P. Popescu is with University Politehnica of Bucureşti, Computer Science and Engineering Department, Bucureşti, Romania (email: george.popescu@cs.pub.ro)

This work has been supported by the Spanish Government (Minesterio de Economa y Competitividad, Fondo Europeo de Desarrollo Regional, FEDER) by means of the projects COSAIF, Connectivity as a Service: Access for the Internet of the Future (TEC2012-38754-C02-01), and ADVICE, Dynamic provisioning of connectivity in high density $5 \mathrm{G}$ wireless scenarios (TEC201571329-C2-1-R) system level simulations, which usually make several assumptions regarding resource modeling, and how the various access elements interfere with each other.

In order to appropriately model the access network behavior, two main aspects need to be considered: (1) the network geometry; and (2) the network load. In cellular networks, the deployment geometry is typically taken into account by means of the interference factor, f-factor [6]. While most of the available works put considerable effort on using appropriate propagation models [7] [8], little attention is paid to the network load, which is usually assumed constant. Opposed to that, some other studies, such as [9], have discussed the impact of these network load assumptions on the access performance, since it modulates the interference. Hence, an accurate evaluation of a particular access policy would require considering the evolution of network load, which implies the precise evaluation of the mutual interference resulting from a particular resource allocation. In this sense, network models for interference coupled systems usually lead to complex optimization problems that cannot be generally solved in a closed form [10]. In this work, we propose two different approaches to mimic the network behavior (resource assignment) for system level simulations, considering both its geometry and the corresponding load fluctuation. The first one exploits heuristic techniques, in particular a Simulated Annealing (SA) algorithm. In the second approach, by taking some simplifications, the underlying problem can be transformed into a generalized geometric program, which, in turn, can be solved by exploiting convex optimization techniques. Although this approach has already been used in resource allocation problems, for instance [11], their scope was not the same and the corresponding problem formulation was therefore different.

\section{SYSTEM MODEL}

We consider a scenario with of a set of users $\mathcal{I}$ and LTE cells $\mathcal{B}$. In this single carrier LTE system, cells share the system resources (i.e. physical resource blocks), so that all base stations, but the serving one, cause interference. We also assume that access selection has been already completed, and users are attached to the cells with highest received signal level (i.e. highest RSRP).

We define the function $\beta$ to identify the base station a user $i$ is connected to, $\beta(i) \in \mathcal{B}$. We also define the set of users connected to a particular cell $k$ as $\mathcal{U}(k) \subseteq \mathcal{I}$. Besides, for each user-cell pair $(i, k)$ we denote $\gamma_{i, k}$ as the power user $i$ receives from base station $k$, while $\Gamma_{i}$ is the one corresponding to the serving cell of user $i$, i.e. $\Gamma_{i}=\gamma_{i, \beta(i)}$. Furthermore, each user 
has a particular traffic demand, $D_{i}$, and her/his serving cell, $\beta(i)$, needs to allocate enough resources $x_{i}$ to satisfy such demand, since we assume non-elastic services.

In order to calculate the amount of resources $x_{i}$ to be allocated to each user, we first need to estimate the spectral efficiency of each connection. We consider that the cells make use of a random scheduler, so that the amount of interference power coming from a cell would be proportional to the load of such cell. A widespread approach, in system level evaluations, is to assume that traffic is evenly distributed within the network, which yields a constant load (CL) for all cells. In this case, the spectral efficiency $\eta^{\mathrm{cl}}$ for a particular user $i$ can be calculated as:

$$
\eta_{i}^{\mathrm{cl}}=\log _{2}\left(1+\frac{\Gamma_{i}}{\sigma^{2}+\sum_{k \in \mathcal{B} / \beta(i)} \frac{L_{k} \gamma_{i, k}}{C}}\right)
$$

where $L_{k}$ is the load of an interfering cell $k \neq \beta(i), \sigma^{2}$ corresponds to the system white additive noise power and $C$ holds for the system capacity. The previous approach offers a relevant advantage: the resources to be assigned can be straightforwardly calculated, since all the parameters are known. On the other hand, if we strictly calculate the cell load that characterizes the particular access selection, we would need to consider the mutual interference (MI) between the various associations. In this case, we can define the spectral efficiency $\eta^{\mathrm{mi}}$ perceived by user $i$ as:

$$
\eta_{i}^{\mathrm{mi}}=\log _{2}\left(1+\frac{\Gamma_{i}}{\sigma^{2}+\sum_{k \in \mathcal{B} / \beta(i)} \frac{\gamma_{i, k}}{C} \sum_{j \in \mathcal{U}(k)} x_{j}}\right)
$$

In this latter case, the solution for the resource assignment problem is more complex, since we can see that the spectral efficiency (and thus the resources to be allocated to the user) depends on the resources granted to other users, $x_{j} \forall j \neq i$.

In order to compare the two approaches, we model the corresponding resource assignment as the following optimization problem, which seeks minimizing the required number of resources, while satisfying the traffic demand for all users (non-elastic traffic):

$$
\begin{array}{ll}
\min & \sum_{i \in \mathcal{I}} x_{i} \\
\text { s.t. } & \sum_{i \in \mathcal{U}(j)} x_{i} \leq C \\
& x_{i} \cdot \eta_{i} \geq D_{i}
\end{array}
$$

where constraint (4) ensures that the cell capacity is not exceeded, while (5) captures the fact that the traffic demand needs to be satisfied for every user. It is worth noting that, in the case of constant load, the problem can be solved directly, by calculating the resources that satisfy the user demand.

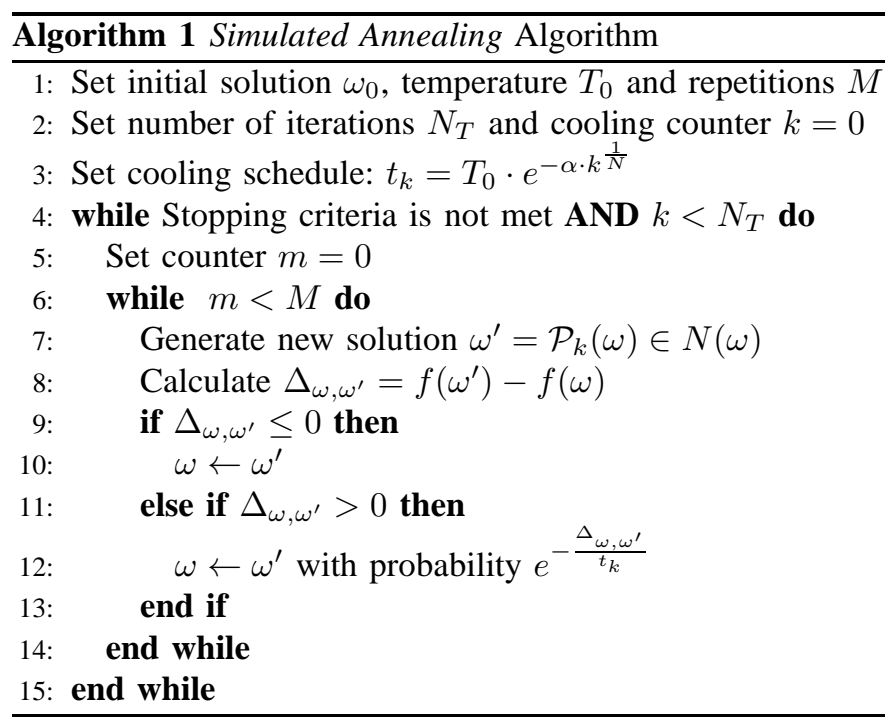

\section{SOLVERS FOR THE MUTUAL INTERFERENCE PROBLEM}

This section depicts the two alternatives that we have used to solve the aforementioned assignment problem, when mutual interference and non-elastic traffic demand are assumed. The first one uses heuristics, in particular Simulated Annealing, to find the optimum assignment. Afterwards, we propose a modification on the formulation that yields a geometric programming problem, which is solved exploiting convex optimization techniques.

\section{A. Simulated annealing}

The SA algorithm follows the generic approach depicted in [12], whose implementation details are described in Algorithm 1. In brief, it contains two nested loops; the first one corresponds to the cooling process, which defines a new temperature, while the inner one implements the thermal equilibrium for each temperature. As can be seen, the algorithm starts by selecting an initial solution $\omega_{0}$ with a cost $f\left(\omega_{0}\right)$, which is calculated assuming that mutual interference does not exist; its value is quite relevant to improve the convergence time. For the cooling procedure, we use an exponential scheme, since it showed an appropriate performance for our scenarios. We start from an initial temperature $T_{0}=500$, and $N_{T}=300$ iterations were used. Furthermore, the algorithm carries out $M=400$ repetitions to look for new neighboring solutions, $N(\omega)$. During this process, neighboring solutions $\omega^{\prime} \in N(\omega)$ are obtained. If the cost evaluated at $\omega^{\prime}, f\left(\omega^{\prime}\right)$, improves the previous one (i.e. $\Delta_{\omega, \omega^{\prime}}<0$ ), this new solution is "approved"; otherwise, it will be approved with a certain probability, which depends on the current temperature.

While the overall process could be used for different problems, the procedure used to seek new neighboring solutions heavily depends on the particular characteristics of the actual problem. We have implemented it as a perturbation of the current solution $\mathcal{P}(\omega)$, defined as $\omega^{\prime}=\omega-g(x ; 0,1) \cdot \frac{\epsilon}{\eta}$, where $g(x ; 0,1)=\frac{1}{\pi\left(1+x^{2}\right)}$ is the standard Cauchy distribution, $\eta$ holds for the spectral efficiency of the current association, and $\epsilon$ is the difference between the traffic demand and the value carried by the current assignment. As can be seen, every new 
solution randomly modulates the current assignment, considering the corresponding capacity demand. This algorithm can indeed solve the problem assuming mutual-interference, but it needs a tailored configuration for each particular scenario to avoid local-minima solutions.

\section{B. Generalized Geometric Programming}

In order to overcome the limitations of the heuristic approach, in this section we propose a transformation of previous formulation, the one with mutual interference, so that it can be solved as a convex problem, exploiting Geometric Programing (GP) [13], which can be generically defined as follows:

$$
\begin{aligned}
\min & f_{0}(x) \\
\text { s.t. } & f_{i}(x) \leq 1, \quad i \forall \mathcal{P} ; \quad g_{i}(x)=1, \quad i \forall \mathcal{M}
\end{aligned}
$$

where $f_{i}$ and $g_{i}$ are generalized posynomials and monomials, respectively. If we take $\log$ at both sides of the previous equations, the problem is transformed into a convex one. Besides, the original variables $x_{i}$ are replaced by $y_{i}=\log x_{i}$.

In the previously proposed problem, both the objective function and the capacity constraint, (3) and (4), respectively, are generalized posynomial functions (in fact, they are linear). On the other hand, the demand constraint (5) is not a posynomial function. However, if we assume that $\operatorname{SINR} \gg 1$, (5) can be simplified, and the original problem can be transformed into a GP one. This assumption would be in fact sensible, considering the high densification of the access network, and advanced cooperation techniques that can be exploited between the various cells, which would yield a higher SINR. By introducing a new slack variable $t_{i}$, the demand constraint can be expressed as:

$$
\begin{aligned}
\frac{\sigma^{2}+\sum_{k \in \mathcal{B} / \beta(i)} \frac{\gamma_{i k}}{C_{k}} \sum_{j \in \mathcal{U}(k)} x_{j}}{\Gamma_{i}} & \leq \frac{1}{t_{i}} \\
t_{i} & \geq e^{\frac{\log 2 \cdot D_{i}}{x_{i}}}
\end{aligned}
$$

We can indeed see that the first new constraint, (8), corresponds to a generalized posynomial function. On the other hand, (9) can be converted into a convex constraint, with a logarithmic transformation [13], i.e. $s=\log t$. Finally, the original resource assignment problem, with mutual interference, can be formulated as a convex problem as follows:

$$
\begin{array}{cl}
\min & \log \sum_{i} e^{y_{i}} \\
\text { s.t. } & \log \left(\frac{\sigma^{2}+\sum_{k \neq \beta(i)} \frac{\gamma_{i k}}{C_{k}} \sum_{j \in \mathcal{U}(k)} e^{y_{j}}}{\Gamma_{i}}\right)+s_{i} \leq 0 \\
& \log (2) \cdot D_{i} / e^{y_{i}}-s_{i} \leq 0 \forall i \\
& \sum_{i \in \mathcal{U}(j)} e^{y_{i}}-C_{j} \leq 0
\end{array}
$$

\begin{tabular}{|c|c|}
\hline LTE layout & 20MHz@2.1GHz \\
\hline Macro layer & $\begin{array}{c}\text { ISD } 500 \mathrm{~m}, 7 \text { tri-sector sites } \\
\text { Max. TX. power } 46 \mathrm{dBm} \\
\text { Antenna Gain } 16 \mathrm{dBi}, 15^{\circ} \text { down-tilt }\end{array}$ \\
\hline Pico layer & $\begin{array}{l}\text { Random Location, } 12 \text { cells per cluster } \\
\text { Max. TX. power } 37 \mathrm{dBm} \\
\text { Omni-antenna, } 5.0 \mathrm{dBi}\end{array}$ \\
\hline$\overline{\mathrm{UE}}$ & DL NF $7 \mathrm{~dB}$, RX. Gain $0 \mathrm{~dB}$ \\
\hline LTE layer & L $(\mathrm{dB})$ as a function of the distance $\mathrm{d}(\mathrm{m})[14]$ \\
\hline $\begin{array}{l}\text { MacronLOS } \\
\text { Macro }_{\text {LOS }}\end{array}$ & $\begin{array}{l}139.1033+39.0864 \cdot\left(\log _{10} d-3\right) \\
36.2995+22 \cdot \log _{10} d \text { if } d<328.42 \\
40 \cdot \log _{10} d-10.7953 \quad \text { if } d>328.42\end{array}$ \\
\hline $\begin{array}{l}\text { Pico }_{\text {LOS }} \\
\text { Picolos }\end{array}$ & $\begin{array}{l}145.48+37.5 \cdot\left(\log _{10} d-3\right) \\
103.8+20.9 \cdot\left(\log _{10} d-3\right)\end{array}$ \\
\hline & LOS probability as a function of the distance $\mathrm{d}(\mathrm{m})$ \\
\hline $\begin{array}{l}\text { Macro } \\
\text { Pico }\end{array}$ & $\begin{array}{l}P_{\mathrm{LOS}}=\min \left(\frac{18}{d}, 1\right) \cdot\left(1-e^{\frac{-d}{36}}\right)+e^{\frac{-d}{36}} \\
P_{\mathrm{LOS}}=0.5-\min \left(0.5,5 \cdot e^{\frac{-156}{d}}\right)+\min \left(0.5,5 \cdot e^{\frac{-d}{30}}\right)\end{array}$ \\
\hline
\end{tabular}

TABLE I: Simulation setting

\section{Simulation Results}

In order to assess the differences between both models, CL and MI, an evaluation of a two-tier urban dense network has been carried out. The first layer corresponds to trisector macro eNodeBs, deployed according to an hexagonal pattern, while the second one comprises pico-cell, which are randomly deployed within the coverage area of the macrocell. Regarding resources, we assume a system bandwidth of $20 \mathrm{MHz}$; hence, 100 resource blocks, of $180 \mathrm{KHz}$ each, are shared.

Table I shows the main simulation parameters. We deploy 100 active users, each of them with a downlink traffic demand of $500 \mathrm{Kbps}$. The analysis is carried out in the central cluster, while the macro-cells in the first interfering tier are assumed to have a constant load, yielding a certain additional interference. In order to ensure the statistical tightness of the results, we have carried out 100 independent runs for every configuration.

In order to assess the impact of considering constant load, Figure 1 depicts the Complementary Cumulative Distribution Function (CCDF) of the relative load in the central cluster assuming a load in the interfering tier of $40 \%$. The results are shown for both macro- and pico-cells, when using CL and MI models; in this later case, we exploited the SA solution. We have assumed two Cell Range Extension (CRE) configurations, 3 and $9 \mathrm{~dB}$. We can clearly see that the load is rather variable, far from the constant value that is assumed in the CL model; this variability is particularly higher for the pico-cells. The impact of the CRE configuration can be clearly seen, since the load of the small cells is much higher when the CRE equals $9 \mathrm{~dB}$. We can thus conclude that the widespread constant-load model could yield inaccurate load values, and that in some cases, especially when the traffic might not be fairly distributed or in highly heterogeneous scenarios, the use of a mutual interference approach shall be preferred.

In Section III we have presented the transformation of the MI problem, so that it can be solved using Geometric Programming techniques; we have used the GPKIT library [15] to do so. In order to assess whether the assumptions and simplifications that were taken are valid, Figure 2 compares the results that were obtained for both the SA and GP solutions. We represent the Cumulative Distribution Function (CDF) of the relative difference on the resources allocated 


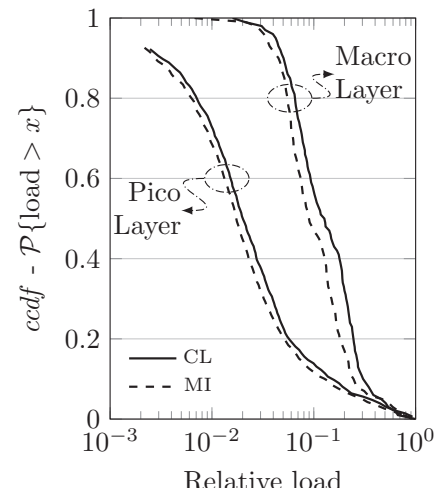

(a) CRE $3 \mathrm{~dB}$

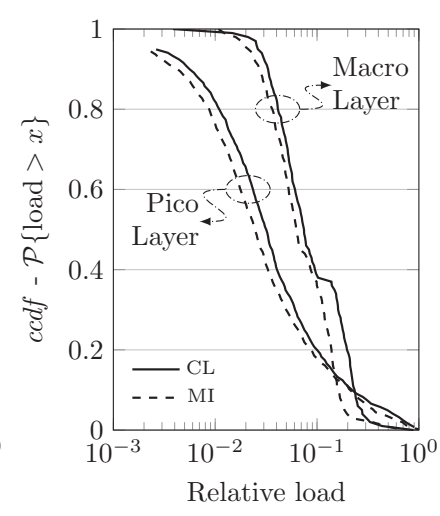

(b) CRE $3 \mathrm{~dB}$
Fig. 1: $c c d f$ of the relative load for the two cell types

for the same scenario instance. Hence, we define $\Delta_{\text {load }}=$ $\frac{\left|\sum_{i \in \mathcal{I}} x_{i}^{\mathrm{sa}}-\sum_{i \in \mathcal{I}} x_{i}^{\mathrm{gp}}\right|}{\sum_{i \in \mathcal{I}} x_{i}^{\mathrm{sa}}}$, where $x_{i}^{s a}$ and $x_{i}^{g p}$ are the resource allocated by cell $i$ for the Simulated Annealing and Geometric Programing solutions, respectively. The results yield that the GP solution exhibits an appropriate performance, especially when the interference induced by the external macro-cells is low. This was expected, since higher interferences would lead to smaller Signal to Interference plus Noise Ratio (SINR), and the simplification that was taken $(\operatorname{SINR} \gg 1)$ would be less precise.

In any case, for a relatively high load (40\%), the difference stays below $10^{-2}$ for almost $90 \%$ of the cases. We can also see that when CRE is higher, the difference between the $\Delta_{\text {load }}$ for the three scenarios is small, due to the increase of the load of small cells, leading to a slightly higher interference.

\section{CONCLUSION}

Most of the system-level analysis of wireless cellular networks deal with two main issues: (1) access selection, and (2) resource assignment. In HetNets, an accurate modeling of the SINR is of utter relevance, since it has a direct impact on the calculation of the amount of resources that need to be allocated to satisfy capacity demands. Several studies assume that all base-stations are evenly loaded, which might lead to inaccuracies in the system model.

In this letter we have proposed a more accurate approach, in which we consider the mutual interference among different associations, which influences the amount of allocated resources. We showed that the resource-allocation problem that can be posed with this mutual-interference model is non-convex, and it thus needs to be solved exploiting heuristic approaches, in particular we used Simulated Annealing. Furthermore, a problem transformation has been proposed, which allows its exact solution by means of Geometric Programming techniques. Making use of a system-level evaluation, we have verified that the closed solution, in spite of the simplification, yields results similar to those obtained with the original problem. In our future work we will exploit the proposed modeling to perform system-level analysis of different access selection solutions for cellular deployments.

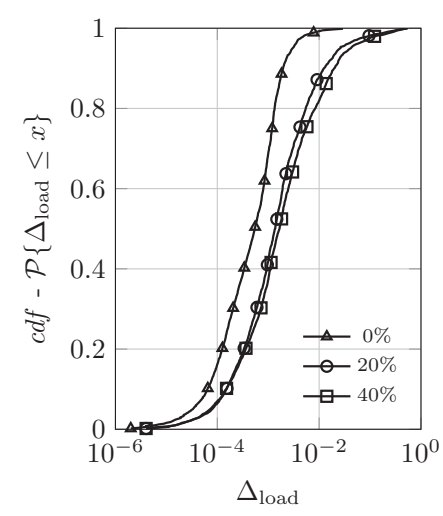

(a) CRE $3 \mathrm{~dB}$

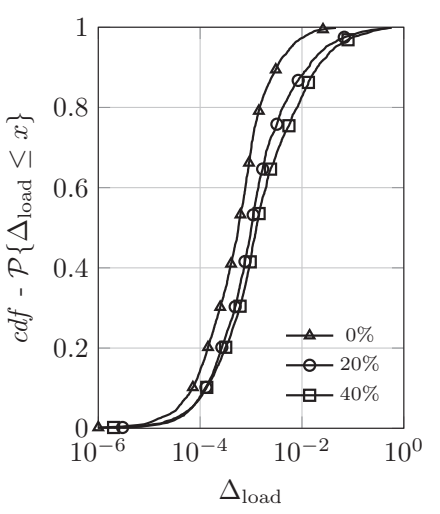

(b) CRE $3 \mathrm{~dB}$
Fig. 2: $c d f$ of the difference of resource assignment with both SA and GP for different loads of interfering cells

\section{REFERENCES}

[1] Cisco, "Cisco visual networking index: Global mobile data traffic forecast update, 20152020 white paper," Feb. 2016. [Online]. Available: http://www.cisco.com/c/en/us/solutions/collateral/serviceprovider/visual-networking-index-vni/mobile-white-paper-c11520862.html

[2] Qualcomm, “The 1000x data challenge," Nov. 2013. [Online]. Available: https://www.qualcomm.com/invention/1000x

[3] N. Bhushan, et al., "Network densification: the dominant theme for wireless evolution into 5G," IEEE Communications Magazine, vol. 52, no. 2, pp. 82-89, February 2014.

[4] F. Boccardi, et al., "Five disruptive technology directions for 5G," IEEE Communications Magazine, vol. 52, no. 2, pp. 74-80, February 2014.

[5] M. Peng, et al., "Fronthaul-constrained cloud radio access networks: insights and challenges," IEEE Wireless Communications, vol. 22, no. 2, pp. 152-160, April 2015.

[6] M. K. Karray, "Study of a key factor for performance evaluation of wireless cellular networks: The f-factor," in Wireless Days (WD), 2009 2nd IFIP, Dec 2009, pp. 1-6.

[7] R. Nasri and A. Jaziri, "On the analytical tractability of hexagonal network model with random user location," Wireless Communications, IEEE Transactions on, vol. PP, no. 99, pp. 1-1, 2016.

[8] M. Castaneda, et al., "On downlink intercell interference in a cellular system," in Personal, Indoor and Mobile Radio Communications, 2007. PIMRC 2007. IEEE 18th International Symposium on, Sept 2007, pp. $1-5$.

[9] M. Davidovic, S. Bjekovic, and I. Tomic, "On the impact of network load on LTE network downlink performance," in 2nd International Conference on Electrical, Electronic and Computing Engineering, IcETRAN 2015, Nov 2015.

[10] H. Boche, S. Naik, and T. Alpcan, "Characterization of convex and concave resource allocation problems in interference coupled wireless systems," Signal Processing, IEEE Transactions on, vol. 59, no. 5, pp. 2382-2394, May 2011.

[11] B. Krasniqi and C. F. Mecklenbrauker, "Maximization of the minimum rate by geometric programming for multiple users in partial frequency reuse cellular networks," in Vehicular Technology Conference (VTC Fall), 2011 IEEE, Sept 2011, pp. 1-5.

[12] D. Henderson, S. H. Jacobson, and A. W. Johnson, Handbook of Metaheuristics. Boston, MA: Springer US, 2003, ch. The Theory and Practice of Simulated Annealing, pp. 287-319. [Online]. Available: http://dx.doi.org/10.1007/0-306-48056-5_10

[13] S. Boyd, et al., "A tutorial on geometric programming," Optimization and Engineering, vol. 8, no. 1, pp. 67-127, 2007. [Online]. Available: http://dx.doi.org/10.1007/s11081-007-9001-7

[14] 3GPP, "Technical specification group radio access network; further advancements for E-UTRA," 3rd Generation Partnership Project (3GPP), TR 36.814. [Online]. Available: http://www.3gpp.org/dynareport/36814.htm

[15] E. Burnell and W. Hoburg, "GPkit software for geometric programming," https://github.com/hoburg/gpkit, 2015, version 0.4.0. 